\title{
SHOCKED MOLECULAR GAS NEAR THE GALACTIC CENTER
}

\author{
J. C. SZCZEPANSKI, ${ }^{1,2}$ P. T. P. HO, ${ }^{2}$ A. D. HASCHICK, ${ }^{3}$ \\ and W. A. BAAN ${ }^{4}$ \\ 1 Massachusetts Institute of Technology, Cambridge,MA \\ ${ }^{2}$ Harvard-Smithsonian Center for Astrophysics, Cambridge,MA \\ 3 Haystack Observatory, Westford, MA \\ 4 Arecibo Observatory, Puerto Rico
}

\begin{abstract}
A $3^{\prime} \times 6^{\prime}$ field centered $\sim 4^{\prime}$ south of the Galactic center and a $\sim 2^{\prime} \times 4^{\prime}$ field east of the Galactic center have been mapped using the $4_{-1} \rightarrow 3_{0} \mathrm{E}$ line $(36.169 \mathrm{GHz})$ of methanol $\left(\mathrm{CH}_{3} \mathrm{OH}\right)$. Line profiles typically consist of broad $\left(\sim 10-20 \mathrm{~km} \mathrm{~s}^{-1}\right)$ components, as well as narrow $\left(\sim 1 \mathrm{~km} \mathrm{~s}^{-1}\right)$,spike-like components. The narrow features are bright and unresolved with $60^{\prime \prime}$ resolution, and are probably due to maser activity. We found maser activity immediately adjacent to the non-thermal "wisp" (SgrA-E) 4' south of SgrA-West, the secondary knot at SgrA-F, and the eastern edge of SgrA-East. Position-velocity diagrams suggest that the molecular material has been shocked to a higher velocity in the same vicinities. We propose that the maser activity, and the shift in velocity south of the Galactic center, heretofore interpreted as an increasingly steep velocity gradient toward the Galactic center, may be due to the impact of a SNR and the resulting shock of the ambient molecular material. The dynamical effects on the molecular gas of such a shock may have implications on gas feeding toward the nucleus.
\end{abstract}

\section{Introduction}

Previous work (Ho et al. 1985) shows that concentrations of dense neutral gas $\left(\mathrm{NH}_{3}\right)$ are on the boundaries of the continuum sources (Fig.1). We mapped a $3^{\prime} \times 6^{\prime}$ field centered $\sim 4^{\prime}$ south of the Galactic center and a $\sim 2^{\prime} \times 4^{\prime}$ field east of the Galactic center using the $4_{-1} \rightarrow 3_{0} \mathrm{E}$ line $(36.169 \mathrm{GHz})$ of methanol $\left(\mathrm{CH}_{3} \mathrm{OH}\right)$. We looked for evidence of supernovae that impact on the neutral gas. We are interested in whether the giant molecular clouds, such as $\mathrm{M}-0.13-0.08$, can be sources of molecular gas to resupply the central neutral ring (cf., Genzel et al. 1985; Güsten et al. 1987).

\section{Observations}

The data were obtained during April and June of 1988. We used the 37-m Haystack telescope with the cooled Q-band maser. We operated in the total power mode, beamswitching every 5 minutes, and sampling a total of $13.3 \mathrm{MHz}\left(110 \mathrm{~km} \mathrm{~s}^{-1}\right)$ bandwidth. Velocity resolution was $0.32 \mathrm{~km} \mathrm{~s}^{-1}$. Pointing was accurate to $\sim 10^{\prime \prime}$ by measuring the nearby SiO maser source WHYDRA. All data has been corrected for atmospheric extinction and elevation-dependent telescope gain variations. Figure 2 shows the measured positions for the two fields centered on sources $\mathrm{C}$ and $\mathrm{G}$ of the 6-cm continuum map of Ho et al. 1985, with integrated intensity contours superposed. 


\section{Results}

Figures $3 a$ and $3 b$ show sample spectra from grids $\mathbf{C}$ and $\mathbf{G}$, respectively. Line profiles typically consist of "broad" ( 10-20 km s$\left.{ }^{-1}\right)$ components, as well as "narrow" $\left(\sim 1 \mathrm{~km} \mathrm{~s}^{-1}\right)$, spike-like components. A weak underlying thermal component is probably present but masked by stronger emission. The "narrow" features are bright and unresolved with 60 " resolution, and are probably due to maser activity. "Broad" components are in good agreement with similar features detected in Sgr B2 and identified as a new type of maser by Morimoto et al. 1985.

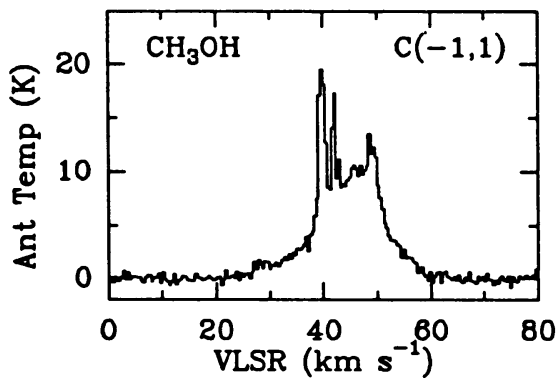

Fig. 3a

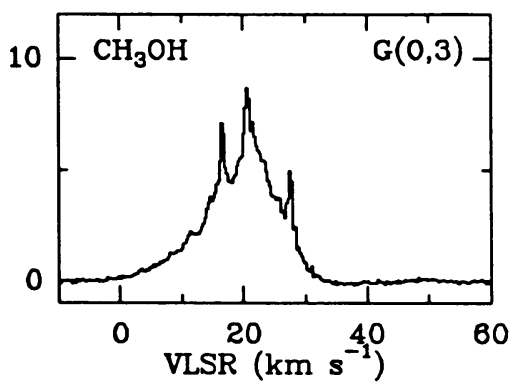

Fig. 3b

Figure 3. - (a) Sample spectrum (Grid C). (b) Sample spectrum (Grid G).

\section{LOCATION OF MASERS: INDICATIVE OF SHOCK AND COMPRESSION?}

The observed spectra show maser activity along the eastern edge of SgrA-East, and immediately adjacent to the "wisp" (SgrA-E) 4' south of SgrA-West and the secondary knot at SgrA-F. These continuum features are non-thermal, polarized, synchrotron emission and may be part of a SNR. Statistical equilibrium calculations (cf. Morimoto et al. 1985) show that collisional excitation can be an effective pumping mechanism for methanol at $T_{K}=80 \mathrm{~K}$. Therefore, spatial correlation of maser activity with the proposed SNR suggests that the population inversion could be established by the impact of the SNR and the resulting shock and compression.

\section{PICKING A KINEMATIC AXIS:}

Figures 5 and 6 show position-velocity diagrams along RA and DEC directions, and at P.A. $= \pm 45$ degrees which are approximately normal and tangent to the "wisp" feature. These figures show that the perceived velocity gradient is in the direction perpendicular to the "wisp" (Fig. 6a), since all three parallel cuts in this direction show a gradient which is not indicated in the other direction (Fig. 6b). Figure 6a further shows that the velocity of the ambient gas shifts abruptly from $\sim 6 \mathrm{~km} \mathrm{~s}^{-1}$ to $\sim 22 \mathrm{~km} \mathrm{~s}^{-1}$ almost exactly at the "wisp" and the secondary knot at SgrA-F. In Figure 6b, the same shift in velocity is observed but with no apparent gradient along the SE-NW direction. This implies that all gas along the tangent strip is subjected to similar dynamical processes. The choice of kinematic axes is significant in that neither preferred axis is directed toward the Galactic center, but rather they are directed nearly perpendicular and parallel to the proposed shock front. Furthermore, the shift in velocity occurs at the position of the proposed SNR. 


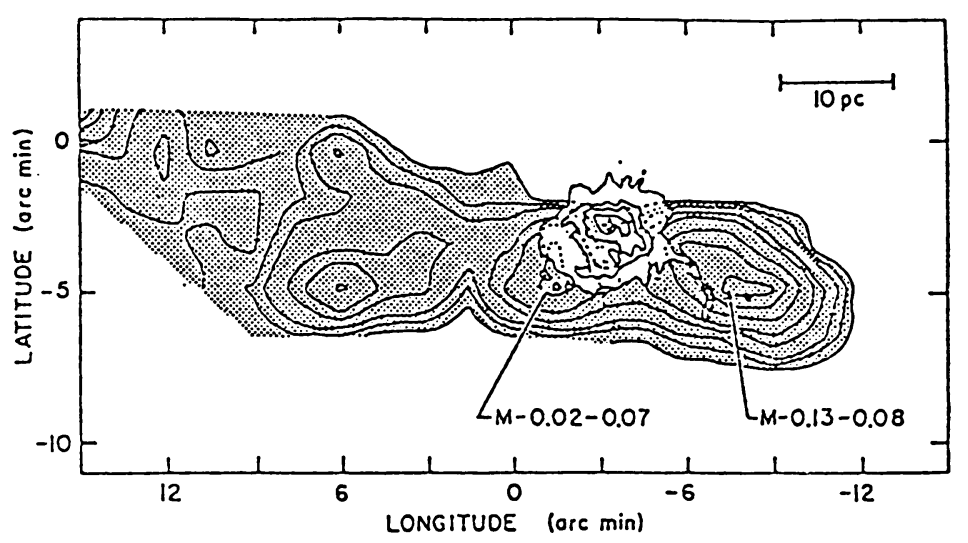

Figure 1. 6-cm continuum map superposed on an $\mathrm{NH}_{3}$ emission map.

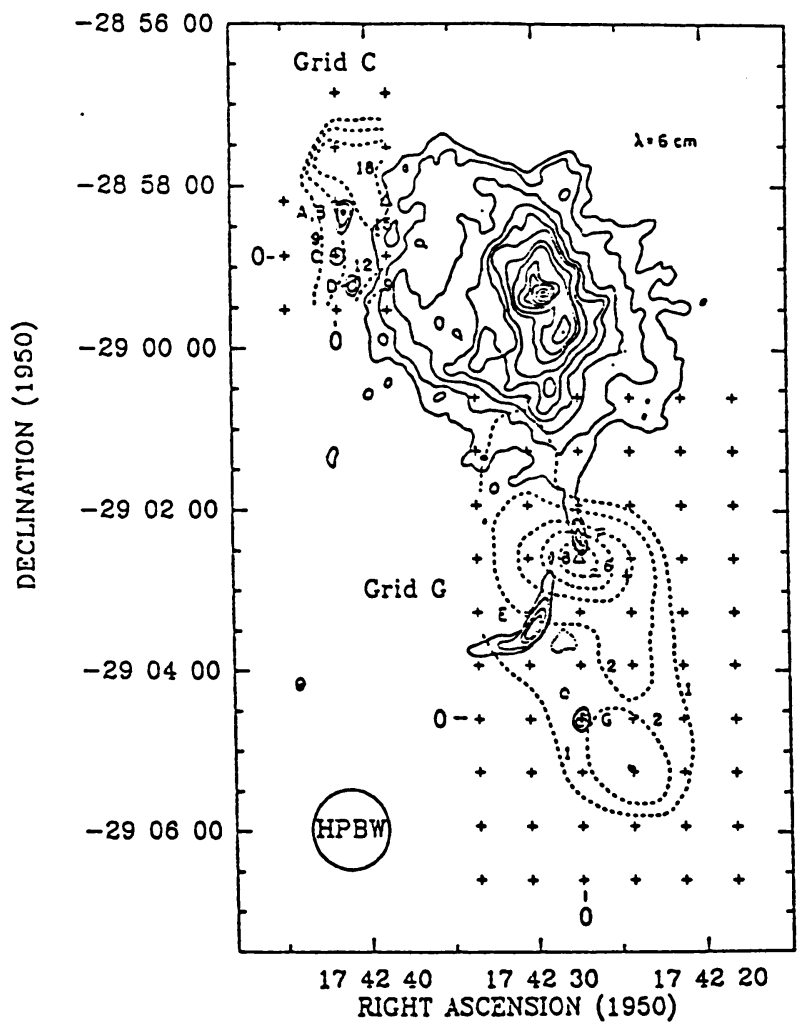

Figure 2. Integrated intensity contours superposed on 6-cm continuum map. Contours are labelled in units of $10 \mathrm{~K}-\mathrm{km} \mathrm{s}^{-1}$. Crosses mark measured positions. Triangles indicate positions of sample spectra in Figure 3. 


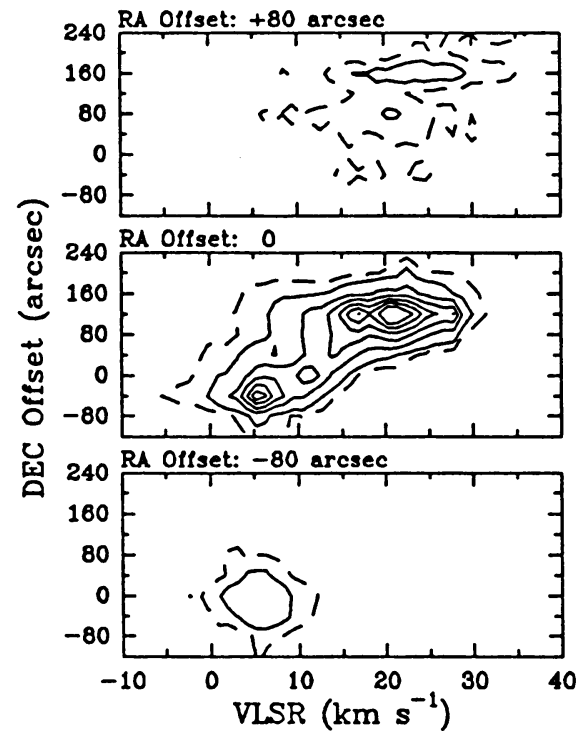

Fig. $5 a$

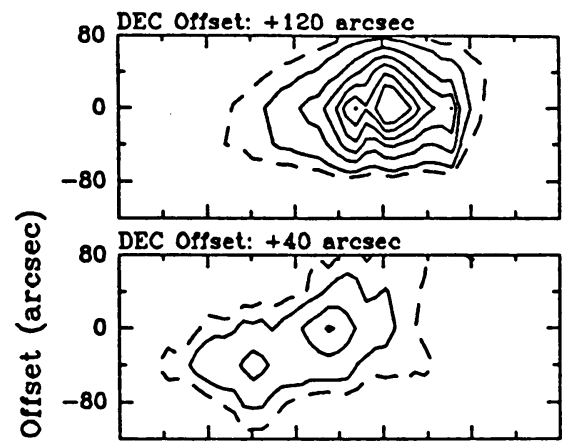

药

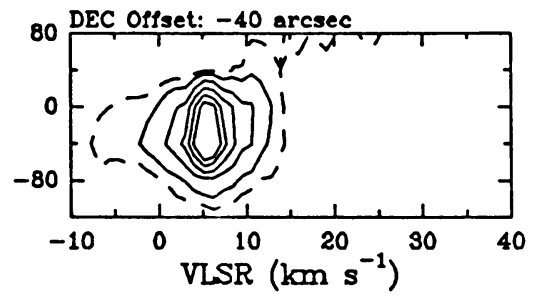

Fig. 5b

Figure 5. Position-velocity diagrams for grid G. Lowest solid contour is $1.0 \mathrm{~K}$. Contour interval is $1.0 \mathrm{~K}$. Dashed contour is $0.5 \mathrm{~K}$. (a) DEC-Velocity diagram. (b) RA-Velocity diagram.

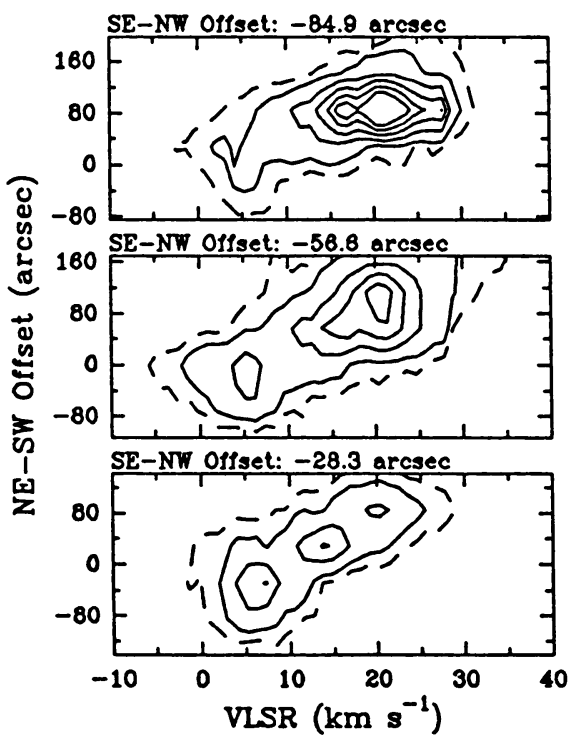

Fig. 6a

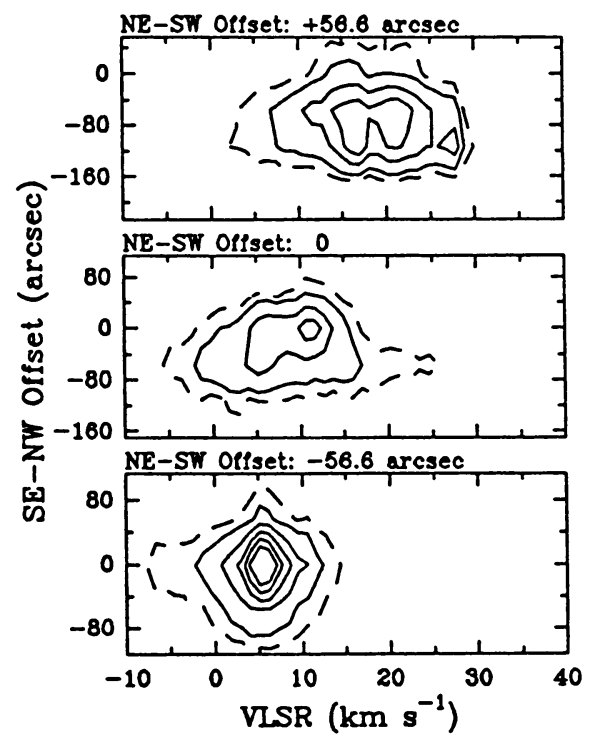

Fig. $6 \mathrm{~b}$

Figure 6. Position-velocity diagrams for grid G. Same contour values as in Figure 5. (a) Cut through data at P.A. $=-45$ degrees. (b) Cut through data at P.A. $=+45$ degrees. 


\section{ROTATION OR SHOCK?}

The velocity structure seen in Figures 5 and 6 suggests rotation as an alternative explanation. Previously, the apparent velocity gradient has been used to infer the mass distribution of the region. However, the present data indicates:

1. A kinematic axis of the gas is not directed toward the Galactic center but is directed perpendicular to the proposed shock front (e.g., Fig. 6a),

2. The velocity shift is accompanied by an increase in linewidth. This may be explained by increased turbulence due to shock processing, but is not explained by a rotation model, and

3. The position of the velocity shift correlates well with the location of maser activity and the purported SNR.

We, therefore, favor the shock explanation for the velocity structure. This shock, if a correct identification, may play a significant role in feeding gas toward the Galactic center (Ho et al. 1985). Similar motions of the neutral gas and the presence of a new $\mathrm{H}_{2} \mathrm{O}$ maser have been reported in this meeting by Okumura-Kawabe et al. 1988. The shocked gas at $20 \mathrm{~km} \mathrm{~s}^{-1}$ may continue toward the Galactic center in the form of a thin streamer (see Ho et al. 1988; these proceedings).

\section{References}

Genzel, R., Watson, D. M., Crawford, M. K. and Townes, C. H. 1985, Ap. J., 297, 766.

Güsten, R., Genzel, R., Wright, M. C. H., Jaffee, D. T., Stutzki, J. and Harris, A. I. 1987, Ap. J., 318, 124.

Ho, P. T. P., Jackson, J. M., Barrett, A. H. and Armstrong, J. T. 1985, Ap. J., 288, 575.

Ho, P. T. P., Jackson, J. M., Armstrong, J. T. and Szczepanski, J. C. these proceedings.

Morimoto, M., Ohishi, M. and Kanzawa, T. 1985, Ap. J. (Letters), 288, L11.

Okumura-Kawabe, S., Ishiguro, M., Fomalont, E. B., Chikada, Y., Kasuga, T., Morita, K. -I., Kawabe, R., Kobayashi, H., Hasegawa, T. and Inoue, M. these proceedings. 